\title{
EFFICACY OF ROBOTIC VERTICALIZATION SYSTEM IN REHABILITATION OF PATIENTS WITH CENTROUS NERVOUS SYSTEM DISORDERS.
}

\author{
Md Resident Vytautas Medutis ${ }^{1}$, PT Donatas Svirskis ${ }^{1}$, Md Resident Jonas Vincas Banaitis ${ }^{1}$, Prof. (HP) MD Alvydas \\ Juocevičius ${ }^{1}$ \\ ${ }^{1}$ Center Of Rehabilitation, Physical And Sports Medicine, Vilnius University Hospital, Santaros Klinikos, Vilnius, Lithuania
}

Intruduction: It is well known that patient verticalization in early stages helps to avoid ortostatic reactions and consequent syncope during very early mobilization. Also early verticalization improves the short-term and long-term functional and neurological outcome. Moreover a new robotic verticalization shows a better results in global motor function, sensory-motor and vestibular system plasticity than physical therapist-assisted verticalization training. Robotic verticalization system plays an important role for patients who have ortostatic reactions in early stages providing the opportunity of early and safe mobilization in order to counteract the negative effects of immobility and accelerating the recovery process with intensive sensomotor stimulation. However, the superiority of robotic verticalization compare to physical therapist assisted training need to be proved for further researches.

Purpose: The aim was to compare effectiveness of classic physical therapy verticalization and training methods with robotic verticalization system (Erigo ${ }^{\circledR}$ Pro) therapy in rehabilitation of patients who suffer from central nervous system disorders.

Methods: The study randomly included 12 patients who have experienced ishemic stroke $(n=6)$, haemoragic stroke $(n=3)$ or spinal cord injury $(n=3)$ from Vilnius University Hospital, Santaros Klinikos, Center of Rehabilitation, Physical and Sports Medicine in-patient unit. Patients were randomly divided in two groups that performed ERIGO training (10 sessions) (G1, $n=6)$ and physiotherapist-assisted verticalization training group (same duration) $(G 2, n=6)$, beyond conventional neurorehabilitation treatment. Study Patient where examined before and after the study. Patients balance was evaluated using Berg balance scale and Tinetti gait and balance scale. Postural control was evaluated using postural assessment scale. Both groups of patients received the same physical therapy sessions. Patients in study group received additional robotic verticalization training $25-30$ minutes a day for 10 sessions. Control group patients received additional 25 - 30 minutes of physical therapist assisted verticalization, passive and active exercises. Data analysis was performed using "Mircrosoft Office Excel 2010" and "SSPS" applications.

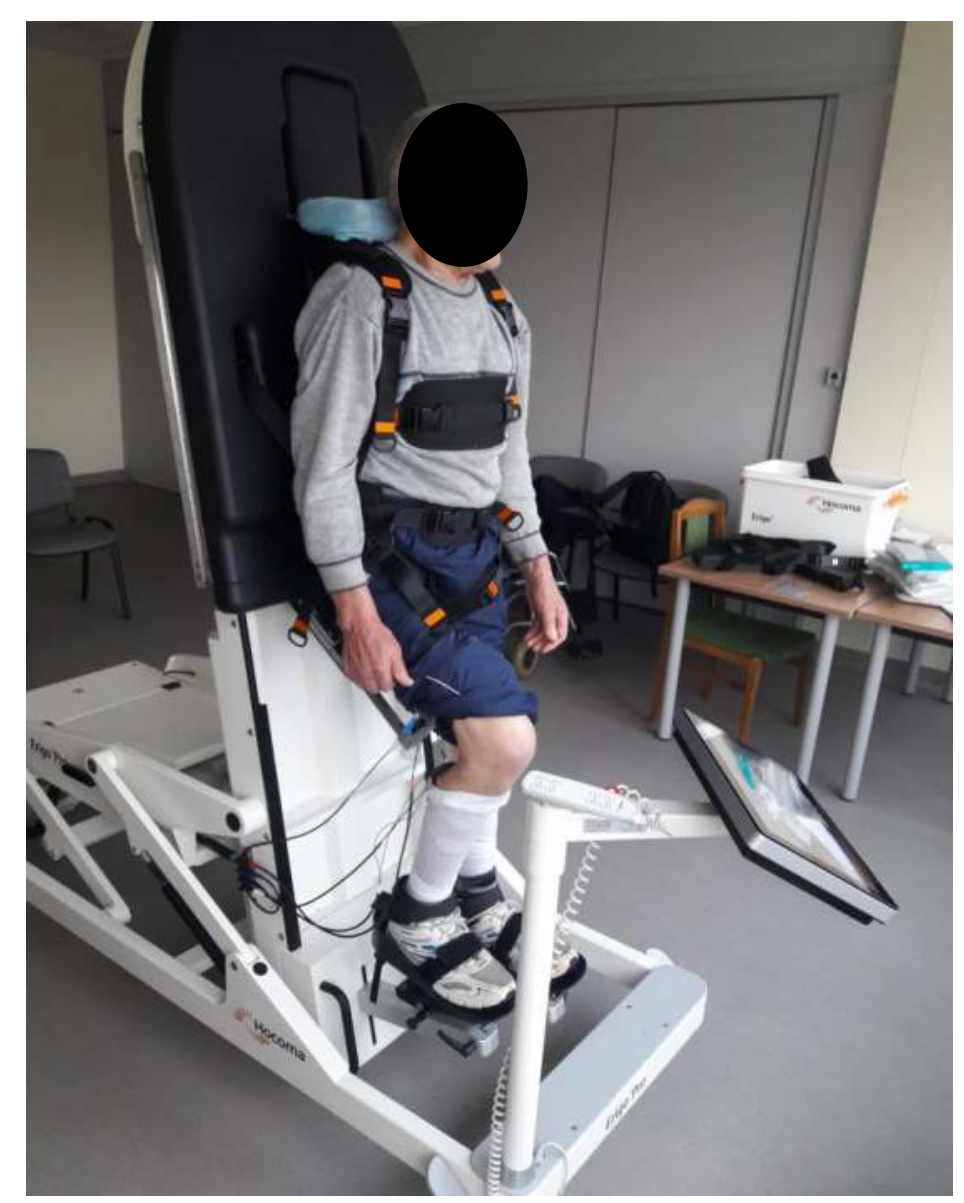

Chart 1: Patient is verticalized with motoric tilt table.
Results: The results of this study have shown that balance and postural control function increased statistically significant in both groups $(p<0,05)$. More positive dynamics were observed in group of patients who received training with robotic verticalization system (chart 2 and chart 3). Patients in study group were able to maintain and train in vertical position sooner that patients in control group.

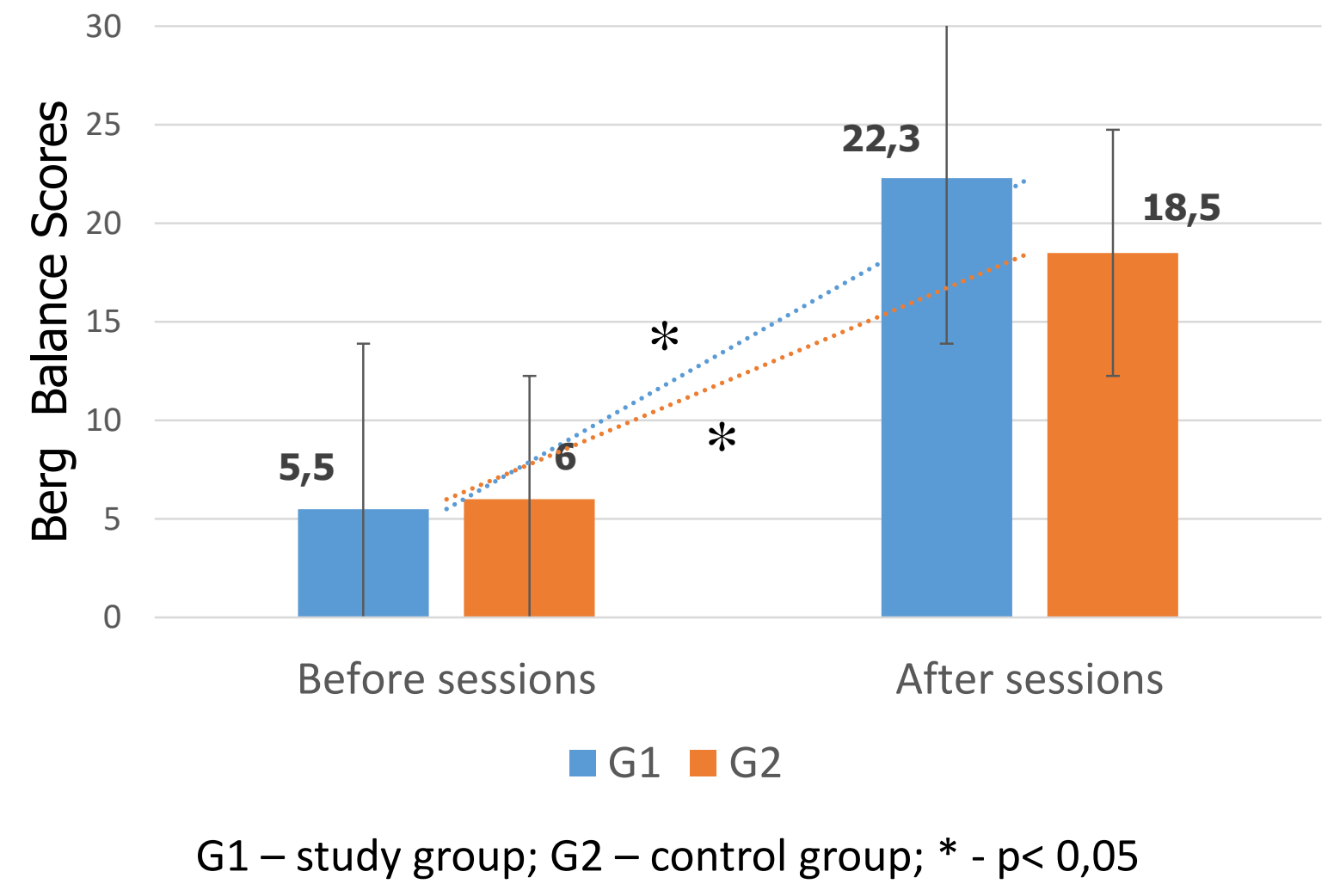

Chart 2: Avarage of Berg balance scores before and after training in both gruops.

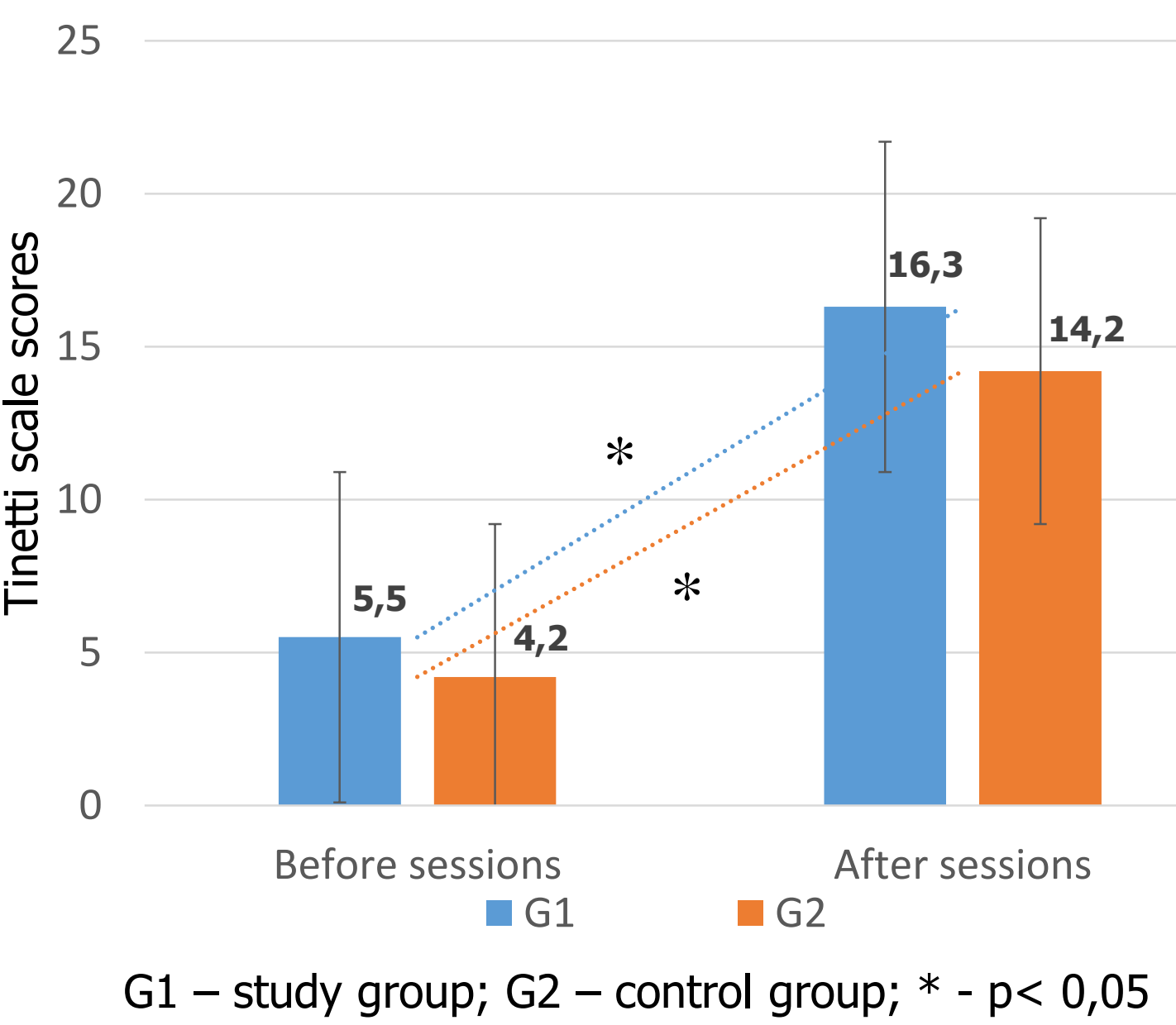

Chart 3 : Avarage of Tinneti scale scores in both groups (G1 and $\mathrm{G} 2$ ), comparison before and after 10 training sessions.

Conclusion: Study have shown that patients in early stage of rehabilitation using robotic verticalization system have slightly higher results in neurological and functional outcome than physical therapist assisted training. Nevertheless, further research with bigger study population are required to prove our findings.



